\title{
MAXIMUM PRINCIPLES FOR A CLASS OF SEMILINEAR ELLIPTIC BOUNDARY-VALUE PROBLEMS
}

\author{
Zhang Hailiang
}

For years it has remained a problem to find suitable functionals satisfying certain maximum principles for solutions of the equation $\Delta u+f(x, u)=0$. In this paper, maximum principles for certain functionals which are defined on solutions of semilinear elliptic equations subject to mixed or Robin boundary conditions are obtained. The principles derived may be used to deduce bounds on important quantities in physical problems of interest.

\section{INTRODUCTION}

In [1], Payne and Stokgold proved the following result:

Let $u$ be a $C^{3}(D)$-solution of

$$
\begin{cases}\Delta u+f(u)=0 & \text { in } D \subset E^{n} \\ u=0 & \text { on } \partial D\end{cases}
$$

If the boundary $\partial D$ has nonnegative mean curvature, then the function

$$
\Phi=|\nabla u|^{2}+2 \int_{0}^{u} f(s) d s
$$

assumes its maximum at a point where $\nabla \boldsymbol{u}=0$.

In [2] Scheafer and Sperb derived maximum principles for certain functions defined for solutions of equations

$$
\Delta u+\lambda \rho(x) f(u)=0
$$

in some region $D \subset E^{2}$ subject to a mixed boundary condition.

In this paper we make use of the classical Hopf maximum principle [5] to derive maximum principles for certain functions defined for solutions of semilinear elliptic equations

$$
\Delta u+f(x, u)=0
$$

\section{Received 3 November 1994}

I would like to thank Professor Ming-Qi Yu for his constant encouragement and helpful criticism.

Copyright Clearance Centre, Inc. Serial-fee code: 0004-9729/95 \$A2.00+0.00. 
in some boundary region $D$ of $E^{n}$, subject to a mixed or Robin boundary condition.

In order to motivate our work, let us first look at the one-dimensional problem

$$
u_{x x}+f(x, u)=0 .
$$

If we multiply (1.2) by $u_{x}$ we get

$$
\frac{1}{2}\left(u_{x}^{2}\right)_{x}+f(x, u) u_{x}=0
$$

that is

$$
\frac{1}{2} u_{x}^{2}+\int_{0}^{u} f(x, s) d s-H(x, u)=\text { constant }
$$

where $H(x, u)$ satisfies: $B_{x}(x, u)=\int_{0}^{u} f_{x}(x, s) d s$. Thus we conclude that the function

$$
P=u_{x}^{2}+2 \int_{0}^{u} f(x, s) d s-2 H(x, u)
$$

is just a constant, where $u$ is a solution of (1.2). It is obvious that $P$ satisfies a maximum principle.

Let $u$ be a solution of (1.1). We look for functions $P$ of the form

$$
P=|\nabla u|^{2}+2 \int_{0}^{u} f(x, s) d s-2 H(x, u),
$$

where $H(x, u)$ satisfies:

$$
H_{, i}(x, u)=\int_{0}^{u} f_{, i}(x, s) d s .
$$

The goal is to find conditions such that (1.5) satisfies a maximum principle.

\section{The main Result and its Proof}

In order to prove the theorems, we first give the following result.

Lemma. Let $u$ be a $C^{3}(D)$ solution of (1.1) with $f \in C^{1}(D \times R), D \subset E^{N}$, $N \geqslant 2$. Then the function $P$ defined by (1.5) takes its maximum either on $\partial D$ or at a critical point of $u$.

Proof: By differentiating (1.5) we obtain

$$
P_{, i}=2 u_{, j} u_{, i j}+2 f u_{, i}
$$


and

$$
\Delta P=P_{, i i}=2 u_{, i j} u_{, i j}+2 u_{, j} u_{, i i j}+2 f \Delta u+2 f_{, i} u_{, i} \cdot
$$

Now

$$
\begin{gathered}
\Delta u=-f, \\
u_{, i i j}=-f_{, j} .
\end{gathered}
$$

This allows us to rewrite (2.2) as

$$
\Delta P=2 u_{, i j} u_{, i j}-2 f^{2} .
$$

From (2.1) and Schwarz's inequality, it follows that

$$
\begin{aligned}
\left(P_{, i}-2 f u_{, i}\right)\left(P_{, i}-2 f u_{, i}\right) & =4 u_{, j i} u, j u, k i u, k \\
& \leqslant 4 u_{, i j} u, i j|\nabla u|^{2} .
\end{aligned}
$$

Consequently, by (2.5) and (2.6), we can write

$$
\Delta P+\frac{L_{k} P_{, k}}{|\nabla u|^{2}} \geqslant 0
$$

where

$$
L_{k}=2 f u_{, k}-\frac{1}{2} P_{, k} .
$$

Hopf's first maximum principle [3] implies the Lemma.

REMARK. One could prove the same result for

$$
P=g(u)|\nabla u|^{2}+2 \int_{0}^{u} f(x, s) g(s) d s-2 H(x, u)
$$

under suitable assumptions on $g(u)$, as shown in [4].

2.1 MIXED BOUNDARY CONDITIONS.

THEOREM 2.1. Let $u$ be a $C^{3}(D)$-solution of the problem

$$
\begin{cases}\Delta u+f(x, u)=0 & \text { in } D \\ u=0 \quad \text { on } \Gamma_{1}, \frac{\partial u}{\partial u}=0 & \text { on } \Gamma_{2}, \Gamma_{1} \cup \Gamma_{2}=\partial D\end{cases}
$$


where $f \in C^{1}(D \times R), D$ is a convex damian in $E^{2}$ and $\frac{\theta u}{\partial n}$ denotes the outward normal derivative. Then the function $P$ defined by (1.5) takes its maximum at a critical point of $u$.

Proof: We shall show that $P$ cannot attain its maximum on $\partial D$ unless it is attained at a critical point of $u$ which is on $\Gamma_{2}$.

Suppose that $P$ takes its maximum at $M \in \Gamma_{1}$. Then $M$ cannot be a critical point of $u$. Since $u=0$ on $\Gamma_{1}$, we have $|\nabla u|=\left|\frac{\partial u}{\partial n}\right|$ and

$$
\frac{\partial P}{\partial n}=2 u_{n} u_{n n}+2 f u_{n}
$$

where $u_{n}$ denotes the outward normal derivative. By introducing normal coordinates in the neighbourhood of the boundary, we can write

$$
\Delta u=u_{n n}+k u_{n}=-f,
$$

where $k$ denotes the curvature of the boundary. Thus it follows that

$$
\frac{\partial P}{\partial n}=-2 k u_{n}^{2}
$$

and since $D$ is convex, $\frac{\partial P}{\partial n} \leqslant 0$ at $M$. This contradicts Hopf's second maximum principle [5].

We now suppose that $P$ takes its maximum at $M \in \Gamma_{2}$ and that $M$ is not a critical point of $u$. Since $\frac{\theta u}{\partial n}=0$ on $\Gamma_{2}$, we have $|\nabla u|=\left|\frac{\partial u}{\partial s}\right|$ and

$$
\frac{\partial P}{\partial n}=2 u, u_{e n}
$$

where $u_{\text {s }}$ denotes the tangential derivative of $u$. In terms of normal coordinates in the neighbourhood of the boundary, we have

$$
u_{e n}=u_{n}-k u_{s}
$$

so that on $\Gamma_{2}$

$$
\frac{\partial P}{\partial n}=-2 k u^{2}
$$

Thus we again arrive at a result contradicting the second maximum principle when $D$ is convex. The Lemma, together with our calculation above, yields Theorem 2.1.

REMARK. Theorem 2.1 is also valid for $n>2$. In this regard, see [4]. 
2.2 ROBIN BOUNDARY CONDITIONS.

TheOREM 2.2. Let $u$ be a $C^{3}(D)$-solution of the problem

$$
\begin{aligned}
& \Delta u+f(x, u)=0 \text { in } D, \\
& \frac{\partial u}{\partial n}+a u=0 \text { on } \partial D, \alpha>0,
\end{aligned}
$$

where $D$ is a convex plane domain, $f \in C^{1}(D \times R)$ and $f>0$. Then $P$, given by (1.5), takes its maximum at a critical point of $u$.

Proof: We shall find that $P$ cannot attain its maximum on $\partial D$.

We write

$$
P=\left(u_{n}^{2}+u_{s}^{2}\right)+2 \int_{0}^{u} f(x, s) d s-2 H(x, u)
$$

and compute

$$
\frac{\partial P}{\partial n}=2\left(u_{n} u_{n n}+u_{s} u_{s n}\right)+2 f u_{n} .
$$

By introducing normal coordinates, we can write

$$
\Delta u=u_{n n}+k u_{n}+u_{s s}=-f,
$$

which together with (2.12) and (2.13), results in

$$
\frac{\partial P}{\partial n}=-2 \alpha^{2} k u^{2}+2 \alpha u u_{s,}-2(\alpha+k) u_{s}^{2} .
$$

Now suppose that $P$ takes its maximum at $M$ on $\partial D$. Then at $M$

$$
\frac{\partial P}{\partial s}=0
$$

where

$$
\begin{aligned}
\frac{\partial P}{\partial s} & =2\left(u_{n} u_{n s}+u_{s} u_{s s}\right)+2 f u_{s} \\
& =2 u_{s}\left(\alpha^{2} u+u_{s s}+f\right) .
\end{aligned}
$$

Either $u_{\mathbb{a}}=0$ or the expression in the braces vanishes at $M$.

CASE 1. Suppose $u_{\mathbb{A}} \neq 0$ at $M$. In this case

$$
u_{g,}=-\left(\alpha^{2} u+f\right) \text {. }
$$

By virtue of the fact that $f>0$, it follows from (1.1) and (2.13) that $u \geqslant 0$ in $D \cup \partial D$. Hence from (2.16), we have $u_{, s} \leqslant 0$ and from (2.14), $\frac{\partial P}{\partial n} \leqslant 0$ at $M$. 
CASE 2. Suppose $u_{s}=0$ at $M$. Under the assumption that $P$ takes its maximum at $M$ on $\partial D$, we know that $P_{s s} \leqslant 0$, where

$$
P_{s,}=\frac{\partial^{2} P}{\partial s^{2}}=2 u_{s s}\left(\alpha^{2} u+u_{s s}+f\right)
$$

Hence, either

(i) $u_{s s} \geqslant 0$ and $\alpha^{2} u+u_{s s}+f \leqslant 0$,

or

(ii) $u_{s s} \leqslant 0$ and $\alpha^{2} u+u_{s s}+f \geqslant 0$.

In case (i), because $f>0$, then $u \geqslant 0$ in $D \cup \partial D$. Under these conditions (i) is impossible, since if $u_{s s} \geqslant 0$, then

$$
\alpha^{2} u+u_{s}+f \geqslant 0
$$

Thus we conclude that (ii) holds, that is $u_{a s} \leqslant 0$. From (2.14) we again deduce that $\frac{\theta P}{\partial n} \leqslant 0$ at $M$. Therefore, by the second maximum principle [5], we conclude that $P$ cannot take its maximum at $M$ on $\partial D$.

The Lemma, in conjunction with our calculations above, yields Theorem 2.2.

REMARK. Theorem 2.2 can be extended to nonlinear boundary conditions $\frac{\theta u}{\partial n}+\sigma(u)=$ 0 , under suitable assumptions on $\sigma(u)>0$.

\section{ConCluding Remarks}

Obviously the principles and applications in $[1,4]$ are covered when $f(x, u)=f(u)$ here. One may give extensions of the maximum principles for a uniformly elliptic equation $L u+f(x, u)=0$ under suitable assumptions, as shown in [4].

Let us now consider a simple illustration in which we determine a bound for the gradient of the solution of a nonlinear Dirichlet problem at any point in the plane bounded domain, in terms of the maximum value of the solution function, the solution function and the diameter of the domain. Let $u$ be a positive $C^{3}(D)$-solution of the problem

$$
\begin{cases}\Delta u+4 u-\left(x_{1}^{2}+x_{2}^{2}\right) \exp \left(a^{2}-x_{1}^{2}-x_{2}^{2}\right)=0 & \text { in } D \\ u=0 & \text { on } \partial D\end{cases}
$$

where $D=\left\{x=\left(x_{1}, x_{2}\right)|| x \mid<a\right\}$. With

$$
f(x, u)=4 u-\left(x_{1}^{2}+x_{2}^{2}\right) \exp \left(a^{2}-x_{1}^{2}-x_{2}^{2}\right),
$$


it follows from Theorem 2.1 that

$$
\begin{aligned}
& |\nabla u|^{2}+2 \int_{0}^{u}\left(4 s-\left(x_{1}^{2}+x_{2}^{2}\right) \exp \left(a^{2}-x_{1}^{2}-x_{2}^{2}\right)\right) d s-2 H(x, u) \\
\leqslant & \max _{D \cup \theta D}\left[2 \int_{0}^{u}\left(4 s-\left(x_{1}^{2}+x_{2}^{2}\right) \exp \left(a^{2}-x_{1}^{2}-x_{2}^{2}\right)\right) d s-2 H(x, u)\right]
\end{aligned}
$$

or

$$
\begin{aligned}
|\nabla u|^{2} & \leqslant \max _{D \cup \theta D}\left[4 u^{2}-2\left(x_{1}^{2}+x_{x}^{2}\right) \exp \left(a^{2}-x_{1}^{2}-x_{2}^{2}\right) u\right] \\
& -\left[4 u^{2}-2\left(x_{1}^{2}+x_{2}^{2}\right) \exp \left(a^{2}-x_{1}^{2}-x_{2}^{2}\right) u\right] .
\end{aligned}
$$

From (3.2), we have

$$
|\nabla u|^{2} \leqslant 4\left(u_{M}^{2}-u^{2}\right)+2 a^{2} e^{a^{2}} u_{M},
$$

where $u_{M}$ is the maxmum value of $u$ in $D \cup \partial D$.

\section{REFERENCES}

[1] L.E. Payne and I. Stakgold, 'Nonlinear problems in nuclear reactor analysis', in Proceedings of the Conference on Nonlinear Problems in Physical Sciences and Biology, Lecture Notes in Math. 322 (Springer-Verlag, Berlin, Heidelberg, New York, 1972), pp. 298-307.

[2] P.W. Schaefer and R.P. Sperb, 'Maximum principles and bounds in some inhomogeneous elliptic boundary value problems', SIAM J. Math. Anal. 8 (1977), 871-878.

[3] M.H. Protter and H.F. Weinberger, Maximum principles in differential equations (Prentice-Hall, Englewood Cliffs, N.J., 1967).

[4] R.P. Sperb, Maximum principles and their applications (Academic Press, New York, 1981).

[5] E. Hopf, 'A remark on linear elliptic differential equations of the second order', Proc. Amer. Math. Soc. 3 (1952), 791-793.

Department of Mathematics

Shanxi University

Taiyuan 030006

The People's Republic of China 